\title{
Redox Potential Control by Drug Binding to Cytochrome P450 3A4
}

\author{
Supplemental Data
}

\author{
Aditi Das $*$, Yelena V. Grinkova ${ }^{\star}$ and Stephen G. Sligar ${ }^{*}$ \\ *Departments of Biochemistry and Chemistry and the Beckman Institute \\ for Advanced Science and Technology, University of Illinois Urbana- \\ Champaign, Urbana, Illinois 61801
}

Corresponding Author: Stephen G. Sligar, Department of Biochemistry, 116 Morrill Hall, University of Illinois, 505 South Goodwin Avenue, Urbana, IL 61801

Phone: 217 244-7395, Fax: 217-265-4073, Email: $\underline{\text { s-sligar@uiuc.edu }}$ 


\section{MATERIALS}

Sodium cholate, testosterone (TS), bromocriptine (BC), erythromycin (ERY) and Amberlite (XAD-2) were purchased from Sigma. CHAPS is from Anatrace, Inc. (Maumee, OH), Emulgen 913 from Karlan Research Products Corp. (Santa Rosa, CA); and POPC (1-Palmitoyl-2-Oleoyl-sn-Glycero-3-Phosphocholine) from Avanti Polar Lipids Inc. (Alabaster, AL). All other chemicals ( $>$ ACS Grade) are purchased from Fisher and were used without further purification. The electrodes are from Bioanalytical systems Inc.

\section{EXPERIMENTAL PROCEDURES}

Expression and Purification of CYP3A4 Nanodiscs: Cytochrome P450 3A4 (CYP3A4) with a C-terminal histidine tag was expressed from the NF-14 construct in the PCWori+ vector as previously described. ${ }^{1-4}$ The CYP3A4 in NF-14 pCW Ori+ vector was a generous gift from Dr. F. P. Guengerich (Vanderbilt University, Nashville, TN). CYP3A4 was expressed and purified from Escherichia coli as previously described with minor modifications. ${ }^{1-4}$

Human CYP3A4 was assembled into Nanodiscs using the membrane scaffold protein MSP1D1(-). ${ }^{5}$ MSPID1(-) is MSP1D1 with the poly (histidine) tag removed as described previously. ${ }^{6}$ Briefly, the purified CYP3A4 from the E. coli expression system in $0.1 \%$ Emulgen 913 was mixed with the disk reconstitution mixture containing MSP1D1(-), POPC, and sodium cholate present in 1:65:130 molar ratios. The detergents (cholate and emulgen) were removed by treatment with Amberlite (XAD-2), to initiate the self assembly process. The resultant mixture was then purified using Ni-NTA column to remove the empty Nanodiscs followed by size exclusion chromatography to obtain homogenous CYP3A4-Nanodiscs. The result of this self-assembly reaction is monomeric CYP3A4 incorporated into a $10 \mathrm{~nm}$ discoidal POPC bilayer stabilized by the encircling amphipathic membrane scaffold protein belt. The CYP3A4-Nanodiscs were prepared in a substrate-free form and kept at $4^{\circ} \mathrm{C}$. For long term storage, the preparations were flash frozen and stored in $-80^{\circ} \mathrm{C}$ in the presence of $10 \%$ glycerol. Expression and purification of CYP3A4-Nanodisc was evaluated by SDS-PAGE as described before. ${ }^{4 \mathrm{~b}}$

Characterization of CYP3A4 Nanodiscs: The CYP3A4-Nanodisc concentration was measured by UV-VIS spectroscopy using a CARY BIO 300 spectrophotometer as described previously. ${ }^{4}{ }^{6}$ CYP3A4-Nanodisc were in $100 \mathrm{mM}$ potassium phosphate buffer $\left(\mathrm{pH}\right.$ 7.4) for the spectroelectrochemical titrations. Titrations were performed at $\sim 25^{\circ} \mathrm{C}$ using CYP3A4-Nanodiscs at a concentration of $\sim 10-15 \mu \mathrm{M}$.

The CYP3A4-Nanodiscs were evaluated for binding to the substrate bromocriptine (BC) before and after every spectroelectrochemical titration to test the functional integrity of the protein used for the redox titrations.

The high spin fraction of CYP3A4-ND was calculated using a MATLAB subroutine which was used to deconvolute the observed spectra into its high spin and low spin components. ${ }^{6}$ The basis spectra used for this deconvolution are obtained for high spin (bromocriptine bound) and low spin CYP3A4-ND at low temperatures. The high spin fraction of substrate free CYP3A4-ND was found to be 11-13\%. On binding 
testosterone and bromocriptine, the high spin content increased to 92-95\%. While on addition of erythromycin, the high spin content was calculated to be $22 \%$.

Figure 1S shows that the Type I spectrum obtained on binding bromocriptine (10 $\mu \mathrm{M})$ testosterone $(200 \mu \mathrm{M})$ and erythromycin $(200 \mu \mathrm{M})$ to CYP3A4-Nanodisc. The spin conversion is higher than $90 \%$ for testosterone and bromocriptine, confirming the structural and functional homogeneity of CYP3A4 in Nanodiscs. ${ }^{4,6}$

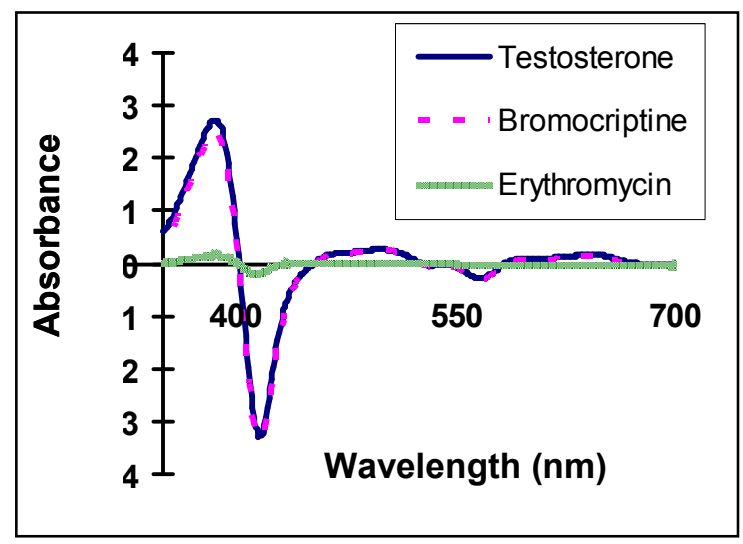

Figure 1S. Difference spectra of CYP3A4 in Nanodiscs where $200 \mu \mathrm{M}$ testosterone (blue solid line), $10 \mu \mathrm{M}$ bromocriptine (pink dotted line) and $200 \mu \mathrm{M}$ erythromycin (green slash line) are shown. On binding testosterone, bromocriptine and erythromycin, the spectra is Type I, indicating generation of high spin iron. The difference spectra are obtained by subtracting the substrate free CYP3A4-Nanodisc spectrum.

Spectroelectrochemical Titrations: The redox titrations used a in-house designed cuvette which was assembled in the anaerobic Cox glove box. The cuvette was sealed with air tight rubber septa through which the reference, the gold working electrode and the gas tight Hamilton needle for titration were inserted. A small magnetic stir bar was placed at the bottom of the cuvette to mix the reagents. The temperature was maintained at $\sim 25^{\circ} \mathrm{C}$.

The titration buffer and the mediators were deoxygenated by flushing it with ultrapure argon while stirring. The protein samples were typically $1.3 \mathrm{~mL}$ with typical concentration of $10-15 \mu \mathrm{M}$. The following redox mediators were introduced prior to titration - methyl viologen $(-430 \mathrm{mV})$, benzyl viologen $(-311 \mathrm{mV})$, 2-hydroxy-1,4naphthoquinone $(-145 \mathrm{mV})$ and anthraquinone 2-sulfonate $(-230 \mathrm{mV})$ at a final concentration of $2 \mu \mathrm{M}$ of each mediator. The mediator concentration was optimized to obtain a balance between the time required to reach equilibrium and to avoid possible mediator-protein interactions. Solutions of CYP3A4-Nanodisc and the mediator mixture were deoxygenated under the flow of argon gas for several minutes and reduced by addition of a small excess of anaerobically prepared dithionite solution with the concentration determined using a molar absorption coefficient $\varepsilon_{315}=8.05 \mathrm{mM}^{-1} \mathrm{~cm}^{-1}$. The reduced protein spectrum was recorded to confirm complete reduction. Absorption spectra $(350-800 \mathrm{~nm})$ were measured on a Cary 3 spectrophotometer (Varian). The protein solutions were titrated electrochemically using sodium dithionite as reductant and potassium ferricyanide as an oxidant. $^{8}$ After each addition of a small aliquot of 
oxidant/reductant, the solution was stirred until equilibration (stabilization of the potential) was reached $(\sim 10-15 \mathrm{~min})$ and the spectra was recorded. The titration was continued until the sample solution was maximally oxidized or reduced.

The system was tested on CYP101, a soluble bacterial P450. The redox potential of CYP101 was found to be $-329 \mathrm{mV}$ for substrate free and $-182 \mathrm{mV}$ for substrate bound which close to literature values which for substrate free CYP101 varies from $-330 \mathrm{mV}$ to $300 \mathrm{mV}$ and for substrate bound varies from -173 to $-163 \mathrm{mV}$. ${ }^{9}$. Figure $2 \mathrm{~S}$ shows the redox titration of CYP 101 with and without the substrate.
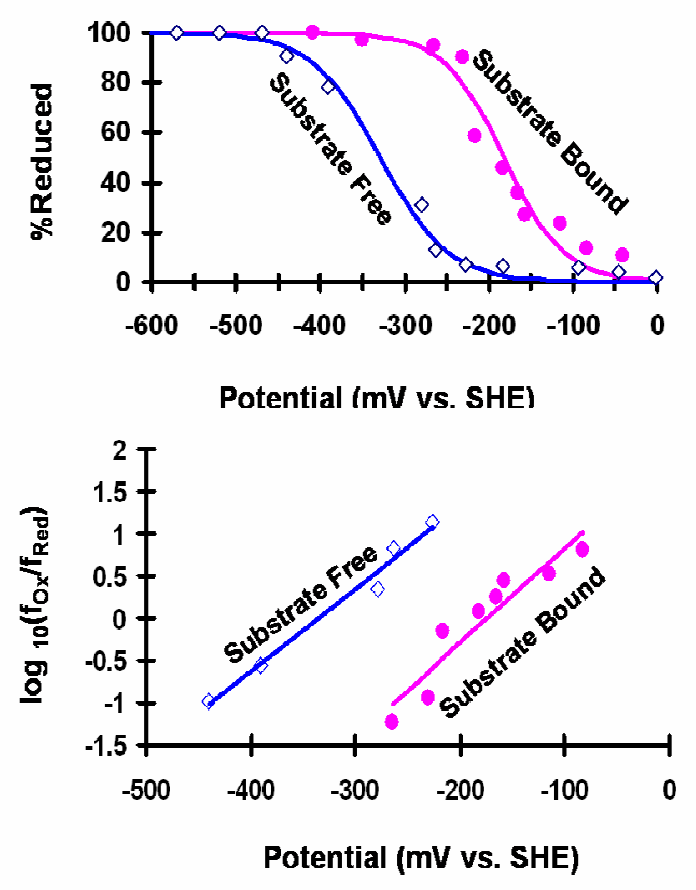

Figure 2S. (Top) Redox titration of substrate-free and camphor bound CYP101. (Bottom) The corresponding Nernst plots used to calculate the redox potential of each species.

In order to probe for interaction of CYP $3 \mathrm{~A} 4$ with the mediators, we have analyzed the spectral changes occurring upon addition of mediator mixture. The spectra of the mixture can be well represented by the sum of the spectra of the components (mediators, proteins, oxidants and reductants). Therefore we could not detect any significant spectrally manifested interaction at the concentrations of components used in this study.

The electrochemical potential was monitored using an Orion $\mathrm{pH} / \mathrm{mV}$ meter (Model SA 720) coupled to a gold electrode and $\mathrm{Ag} / \mathrm{AgCl}$ reference electrode from the bioanalytical systems. Prior to modification, the gold working electrode was cleaned by boiling in $2 \mathrm{M}$ potassium hydroxide for one hour following electrochemical cleaning as described by Katz and Solov'ev ${ }^{10}$. The electrodes were immersed in saturated 4,4'dithiodipyridine solution for $1 \mathrm{~h}$. The electrode system was calibrated using the ferrousferric ammonium sulfate couple $(+675 \mathrm{mV}) .{ }^{11}$ The observed potential was obtained relative to the $\mathrm{Ag} / \mathrm{AgCl}$ reference electrode. Hence they were corrected (using the calibration data for the ferrous-ferric ammonium sulfate solution) to values relative to the 
standard hydrogen electrode (SHE) by addition of $197 \mathrm{mV}$ to the data obtained using the $\mathrm{Ag} / \mathrm{AgCl}$ electrode.

\section{REFERENCES}

1. Domanski, TL, Liu, J, Harlow, GR, and Halpert, JR (1998) Arch. Biochem.Biophys. 350, 223232.

2. Hosea, NA, Miller, GP, and Guengerich, FP (2000) Biochemistry 39, 5929-5939.

3. Gillam, EM, Baba, T, Kim, BR, Ohmori, S, and Guengerich, FP (1993) Arch. Biochem. Biophys. 305, 123-131.

4. (a) Denisov IG, Baas, BJ, Grinkova, YV and Sligar, SG (2007) J. Biol. Chem. 282, 7066-76. (b) Denisov IG, Grinkova, YV, Baas, BJ, and Sligar, SG (2006) J. Biol Chem. 281, 23313-8.

5. Denisov IG, Grinkova, YV, Lazarides, AA, and Sligar, SG (2004) J. Am. Chem. Soc. 126, 3477-3487.

6. Baas, BJ, Denisov, IG , and Sligar, SG (2004) Arch. Biochem. Biophys. 430, 218-228.

7. McKenna, CE, Gutheil, WG and Song, W (1991) Biochimica et Biophysica Acta (BBA) Bioenergetics 1075, 109-117.

8. Dutton, PL (1978) Methods in Enzymology, 54, 411-435.

9. (a) Sligar, SG (1976) Biochemistry, 15, 5399-5406. (b) Martinis SA, Blanke SR, Hager LP, Sligar SG, Hoa GH, Rux JJ, Dawson JH. (1996) Biochemistry, 35, 14530-14536.

10. Katz, EY and Solov'ev, AA, (1990) J. Electroanal. Chem. 291, 171-186.

11. Truman SL (1972) Analytical Chemistry, 44, 6, 1038-1039. 